\title{
Exact controllability of non-Lipschitz semilinear systems
}

\author{
Radosław Zawiski®
}

\begin{abstract}
We present sufficient conditions for exact controllability of a semilinear infinite-dimensional dynamical system. The system mild solution is formed by a noncompact semigroup and a nonlinear disturbance that does not need to be Lipschitz continuous. Our main result is based on a fixed point-type application of the Schmidt existence theorem and illustrated by a nonlinear transport partial differential equation.
\end{abstract}

Mathematics Subject Classification. 37L50, 93B05, 37C25.

Keywords. Exact controllability, nonlinear infinite-dimensional dynamical system, Schmidt existence theorem, fixed point theory.

\section{Introduction}

Controllability of nonlinear systems is a mature subject of research - see $[3,16,18]$ and references therein. In recent years, various applications of fixed point theorems are particularly popular among researchers tackling this problem. These range from classical Banach-Schauder Fixed Point Theorems (FPT) to more specific, such as Nussbaum [15] FPT in [18], Schaefer [22] FPT in [20] or Mönch [13] FPT in [12]. A short survey on fixed point approaches is given in [26].

In most cases, the nonlinearities present in (otherwise linear) systems are regarded as disturbances, in some way influencing the normal operation of a system. The choice of the fixed point-type approach depends on the nature of nonlinearity and the structure of the system itself. The examples of such approach we particularly focus on, are given in $[12,18]$. In the former case, the authors make use of the fact that the system under consideration can be represented as a sum of Lipschitz-type and compact operators, what allows them to apply the Nussbaum [15] fixed point theorem. In the latter case, the authors examine the controllability conditions of a semilinear impulsive mixed Volterra-Fredholm functional integro-differential evolution differential system with finite delay and nonlocal conditions by means of measures of noncompactness and Mönch fixed point theorem [13]. 
Interesting, however, is that although 28 years separate articles [12] and [18], they both contain the assumption of the Lipschitz type of nonlinearity. The author of this article is actually not aware of any example of fixed point theorem application to the problem of exact controllability where the nonlinearity is not Lipschitz in some way. The reason is that with Lipschitz condition comes either computational 'smoothness', which goes back to existence results for initial value problem such as the Picard-Lindelöf theorem, or equicontinuity needed by the Ambrosetti theorem to express the measure of noncompactness.

For this reason, we intentionally drop the Lipschitz condition. In this way, this article combines and expands above results by means of the Schmidt existence theorem, originally developed for the Cauchy problem in Banach spaces [21]. This requires a reformulation of the theorem into the fixed point form. The set of assumptions is discussed and the results follow. In particular, we do not impose any compactness condition. The article is finished with an illustrative example.

\section{Preliminaries}

This section gives the basic definitions and background material. It also defines the notation. If for lemmas or theorems given without reference to a particular source the proof is short and simple, they are immediately followed by the $\square$ sign.

\subsection{On measures of noncompactness, one-sided Lipschitz condition and Schmidt Theorem}

Lemma 2.1. Let $\Xi$ be a normed space and $x, y \in \Xi$. Then the real function $p: \mathbb{R} \rightarrow[0 ;+\infty), p(t):=\|x+t y\|$ is convex.

Corollary 2.2. If for given $t \in \mathbb{R}$ the left-hand side derivative $p_{-}^{\prime}$ of $p$ given by above Lemma exists at point $t$ and the right-hand side derivative $p_{+}^{\prime}$ also exists at point $t$, then the inequality $p_{-}^{\prime}(t) \leq p_{+}^{\prime}(t)$ holds.

Definition 2.3. (One-sided Lipschitz condition) Let $\Xi$ be a Banach space and $x, y \in \Xi$. We define the symbol

$$
[x, y]_{ \pm}:=\lim _{h \rightarrow 0^{ \pm}} \frac{\|x+h y\|-\|x\|}{h}
$$

and say that a function $f: \Xi \rightarrow \Xi$ fulfils one-sided Lipschitz condition (left "-" or right "+", respectively) if there exists a nonnegative constant $M$ such that

$$
[x-y, g(x)-g(y)]_{ \pm} \leq M\|x-y\|
$$

for any $x, y \in \Xi$.

Lemma 2.4. If the limit in Definition 2.3 exists, then $[x, y]_{-}=p_{-}^{\prime}(0)$, $[x, y]_{+}=p_{+}^{\prime}(0)$ and
(a) $[x, y]_{-} \leq[x, y]_{+}$
(b) $\left|[x, y]_{ \pm}\right| \leq\|y\|$ 
(c) $[0, y]_{ \pm}= \pm\|y\|$

(d) $[x, y+z]_{ \pm} \leq[x, y]_{ \pm}+\|z\|$.

Proof. Proofs of points (a)-(c) follow directly from the bracket definition above. We will show only the last one.

1. Consider the case $h<0$ :

$$
\|x+h(y+z)\| \geq\|x+h y\|-\|h z\|=\|x+h y\|+h\|z\|,
$$

and the case $h>0$ :

$$
\|x+h(y+z)\| \leq\|x+h y\|+\|h z\|=\|x+h y\|+h\|z\| .
$$

2. In both estimations in (1) by subtracting $\|x\|$ from both sides and dividing, respectively, by $h<0$ or $h>0$, one obtains

$$
\frac{\|x+h(y+z)\|-\|x\|}{h} \leq \frac{\|x+h y\|-\|x\|}{h}+\|z\| .
$$

3. Going to the limit in (2), the result follows.

Lemma 2.5. Let $\Xi$ be a Banach space, $f: \mathbb{R} \times \Xi \rightarrow \Xi$ and $M$ be a nonnegative constant. Introducing the notation

$$
\begin{aligned}
& \text { (l-) }[x-y, f(t, x)-f(t, y)]_{-} \leq M\|x-y\| \quad \forall x, y \in \Xi \\
& \text { (l) }[x-y, f(t, x)-f(t, y)]_{+} \leq M\|x-y\| \quad \forall x, y \in \Xi \\
& \text { (l) }\|f(t, x)-f(t, y)\| \leq M\|x-y\| \quad \forall x, y \in \Xi
\end{aligned}
$$

the chain of implications $(l) \Rightarrow\left(l_{+}\right) \Rightarrow\left(l_{-}\right)$is true.

Proof. Based on the previous lemma the following estimation holds:

$$
\begin{aligned}
& {[x-y, f(t, x)-f(t, y)]_{-} \leq[x-y, f(t, x)-f(t, y)]_{+} \leq\|f(t, x)-f(t, y)\|} \\
& \quad \leq M\|x-y\| .
\end{aligned}
$$

Lemma 2.6. Let $\Xi$ be a real inner product space. Then

(a) $[x, y]_{ \pm}=\frac{1}{\|x\|}\langle x, y\rangle \quad \forall x, y \in \Xi, x \neq 0$.

(b) Suppose $D \subset \mathbb{R} \times \Xi$ and $f: D \rightarrow \Xi$. Then both conditions

$$
\left(l_{ \pm}\right)[x-y, f(t, x)-f(t, y)]_{ \pm} \leq M\|x-y\| \quad \forall(t, x),(t, y) \in D
$$

are equivalent to

$$
\langle x-y, f(t, x)-f(t, y)\rangle \leq M\|x-y\|^{2} \quad \forall(t, x),(t, y) \in D .
$$

Proof. Proof follows from a straightforward calculation using the Definition 2.3.

Before proceeding further, we recall

Definition 2.7 (Diameter of a set). Let $\Xi$ be a metric space with a metric $\rho$. The diameter of a set $A \subseteq \Xi$ is defined as

$$
\operatorname{diam} A:=\sup _{x, y \in A} \rho(x, y) \leq \infty .
$$

For the case of an empty set, we take $\operatorname{diam} \emptyset=0$.

We can now introduce one of the most commonly used measures of noncompactness (MNC), namely 
Definition 2.8 (Kuratowski measure of noncompactness [11]). For a bounded subset $A$ of a metric space $\Xi$, we call

$$
\alpha(A):=\inf \left\{\delta \geq 0: A \subseteq \bigcup_{i=1}^{n} A_{i} ; \operatorname{diam} A_{i} \leq \delta, i=1, \ldots, n ; n \in N\right\}
$$

the Kuratowski $M N C$.

The Kuratowski MNC has properties given by the following:

Theorem 2.9 (Properties of the Kuratowski $M N C[2,7]$ ). For bounded $A, B \subseteq$ $\Xi$ and $\alpha M N C$, we have
(a) $\alpha(A) \leq \operatorname{diam} A$
(b) if $A \subseteq B$ then $\alpha(A) \leq \alpha(B)$
(c) $\alpha(A \cup B)=\max \{\alpha(A), \alpha(B)\}$
(d) $\alpha(\operatorname{cl} A)=\alpha(A)$ where $\mathrm{cl}$ stands for closure
(e) if $\Xi$ is a normed space and $\operatorname{dim} \Xi=\infty$ then $0 \leq \alpha(B(0,1)) \leq 2$

Additionally, if $\Xi$ is a Banach space, than the following Theorem is true [4]:

Theorem 2.10 For bounded subsets $A, B$ of a Banach space $\Xi$, a constant $m$ and a $M N C \alpha$ there is

(f) $\alpha(A)=0$ if and only if $A$ is relatively compact

(g) $\alpha(A+B) \leq \alpha(A)+\alpha(B)$

(h) $\alpha(m A)=|m| \alpha(A)$

(i) $\alpha(\operatorname{conv} A)=\alpha(A)$ where conv stands for convex hull

In the sequel, to analyse the equicontinuity of a set of functions we will make use of the following items [5].

Definition 2.11 An ordered linear space $Y$ is a space $Y$ on which there is defined a binary relation $\leq$ such that for all $x, y, z \in Y$ the following conditions are satisfied:
(a) $x \leq x$
(b) $x \leq y$ and $y \leq z$ imply $x \leq z$
(c) $x \leq y$ implies $x+z \leq y+z$
(d) $x \leq y$ implies $a x \leq a y$ for all real numbers $a>0$.

Definition 2.12 A wedge $C$ is a nonempty subset of a liner space $Y$ satisfying

$$
a C+b C \subseteq C \quad \forall a, b \in[0, \infty) .
$$

A positive wedge of an ordered linear space $Y$ is the set $Y_{+}$of all elements $x \in Y$ such that $0 \leq x$, where 0 denotes the zero element of $Y$.

We see that $Y_{+}$is a wedge. Conversely, if $C$ is a wedge in a real linear space $Y$, then the binary relation $\leq$ given by

$$
x \leq y \text { if } y-x \in C,
$$

satisfies all conditions in Definition 2.11 for all $x, y, z \in Y$, and in consequence makes $Y$ into an ordered linear space whose positive wedge is exactly $C$. The relation $\leq$ defined by (1) is called the ordering induced by $C$. 
Let $Y$ be a topological linear space. Then $C$ is said to be a normal wedge if for each neighbourhood $W$ of 0 in $Y$ there exists a neighbourhood $V$ of 0 in $Y$ such that

$$
(V-C) \cap(V+C) \subseteq W .
$$

Definition 2.13 Let $M$ be a convex subset of a linear space $X$ and $Y$ be an ordered linear space. Then $f: M \rightarrow Y$ is called a convex function when for all $a \in[0,1]$ and $x, y \in M$ the inequality

$$
f(a x+(1-a) y) \leq a f(x)+(1-a) f(y)
$$

holds. When the order on $Y$ is induced by a wedge $C$, the above can be written as

$$
a f(x)+(1-a) f(y) \in f(a x+(1-a) y)+C .
$$

The following Theorem, taken from [10], is a generalization of the well known Banach-Steinhaus Theorem [19, Theorem 2.5]:

Theorem 2.14 Let $M$ be an open convex subset of a topological vector space $X$ of the second category, let $Y$ be a topological vector space ordered by a normal wedge $C$, and let $\mathcal{F}$ be a pointwise bounded family of continuous convex operators $f: M \rightarrow Y$. Then $\mathcal{F}$ is equicontinuous.

A generalization of Theorem 2.14 to the class of s-convex functions, containing a necessary and sufficient condition of equicontinuity, can be found in [5], and is further developed in [6].

In expressing MNC in function space, a key role is played by the following:

Theorem 2.15 (Ambrosetti [1]). Suppose that $J$ is a compact interval, $\mathcal{F} \subset$ $C(J, E), E$ is a Banach space, $\mathcal{F}$ is bounded and equicontinuous. Then

$$
\alpha(\mathcal{F})=\sup _{t \in J} \alpha(\mathcal{F}(t))=\alpha(\mathcal{F}(J)) .
$$

We also make use of the following:

Definition 2.16 Let $\Xi_{1}$ and $\Xi_{2}$ be metric spaces, $\Phi: \Xi_{1} \rightarrow \Xi_{2}$ be continuous and mapping bounded sets $A \subseteq \Xi_{1}$ onto bounded sets $\Phi(A) \subseteq \Xi_{2}$. If there exists a constant $M \geq 0$ such that for every bounded $F \subset \Xi_{1}$ the inequality

$$
\alpha(\Phi(F)) \leq M \alpha(F)
$$

holds, then $\Phi$ is called an $\alpha$-condensing operator with constant $M$.

The main tool we will use to prove our results is given by $[21,25]$

Theorem 2.17 (Schmidt). Let $X$ be a Banach space and $T, M_{g}, M_{k}$ be reals. Suppose $g, k:[0, T] \times X \rightarrow X$ are continuous, bounded and

(a) $\left[x_{1}-x_{2}, g\left(t, x_{1}\right)-g\left(t, x_{2}\right)\right]_{-} \leq M_{g}\left\|x_{1}-x_{2}\right\| \quad \forall t \in[0, T], \forall x_{1}, x_{2} \in X$

(b) $\alpha(k([0, T], D)) \leq M_{k} \alpha(D) \quad \forall D \subseteq X, D$ bounded. 
Then the initial value problem (IVP)

$$
\left\{\begin{array}{l}
\frac{\mathrm{d}}{\mathrm{d} t} x(t)=g(t, x(t))+k(t, x(t)) \\
x(0)=0
\end{array}\right.
$$

has a solution $x:[0, T] \rightarrow E$.

A function $g$ with properties as above will be called dissipative with constant $M_{g}$, or dissipative with $M_{g}$ for short, and a function $k$ with properties as above will be called condensing with constant $M_{k}$ or condensing with $M_{k}$.

We will use an integral form of (2), as it better suits our needs, that is,

$$
x(t)=\int_{0}^{t} g(s, x(s)) \mathrm{d} s+\int_{0}^{t} k(s, x(s)) \mathrm{d} s, \quad t \in[0, T],
$$

as every solution of (2) is also a solution of (3).

\subsection{On dynamical systems}

From this point onward, we drop the general Banach space setting. Although some of the definitions make sense and the results are true, the Hilbert space setting allows us to obtain more concrete results. Hence, throughout the rest of this paper, $X$ and $U$ are Hilbert spaces which are identified with their duals. For the whole remaining part $J:=[0, T]$ is a compact interval.

We will also use the Sobolev space of vector valued functions

$$
H^{1}(J, X)=W^{1,2}(J, X):=\left\{f \in L^{2}(J, X): \frac{\mathrm{d}}{\mathrm{d} t} f(t) \in L^{2}(J, X)\right\} .
$$

Let $A: D(A) \rightarrow X$ be a densly defined, linear, closed and unbounded operator on which the Cauchy problem of interest is based. Before introducing the Cauchy problem formally, we describe the setting in which it will be considered.

Basic properties of a generator of a strongly continuous semigroup are gathered in the proposition below [17, Theorem 1.2.4]:

Proposition 2.18 Let $(Q(t))_{t \geq 0}$ be a strongly continuous semigroup and let $(A, D(A))$ be its generator. Then

(a) there exist constants $\omega \geq 0$ and $M \geq 1$ such that for every $t \geq 0$ there is

$$
\|Q(t)\| \leq M e^{\omega t},
$$

(b) for every $x \in X$ the function $t \mapsto Q(t) x$ is continuous from $[0, \infty)$ into $X$

(c) for every $x \in X$

$$
\lim _{h \rightarrow 0} \frac{1}{h} \int_{t}^{t+h} Q(s) x \mathrm{~d} s=Q(t) x,
$$

(d) for every $x \in X$ there is $\int_{0}^{t} Q(s) x \mathrm{~d} s \in D(A)$ and

$$
A \int_{0}^{t} Q(s) x \mathrm{~d} s=Q(t) x-x,
$$


(d) for every $x \in D(A)$ there is $Q(t) x \in D(A)$ and

$$
\frac{\mathrm{d}}{\mathrm{d} t} Q(t) x=A Q(t) x=Q(t) A x,
$$

(e) for every $x \in D(A)$

$$
Q(t) x-Q(s) x=\int_{s}^{t} Q(\tau) A x \mathrm{~d} \tau=\int_{s}^{t} A Q(\tau) x \mathrm{~d} \tau .
$$

The operator $A^{*}: D\left(A^{*}\right) \rightarrow X$ is the adjoint of $A$. Important properties of the adjoint are summarized in the following remark [24, Chapter 2.8].

Remark Let $A: D(A) \rightarrow X$ be a densely defined operator with $s \in \rho(A)$. The following holds:

1. If $A$ is closed (as $\rho(A)$ is not empty) we conclude that $A^{*}$ is also closed, densely defined on $X$ and $A^{* *}=A$.

2. There is $\bar{s} \in \rho\left(A^{*}\right)$ and $\left[(s I-A)^{-1}\right]^{*}=\left(\bar{s} I-A^{*}\right)^{-1}$.

3. Let $(Q(t))_{t \geq 0}$ be a strongly continuous semigroup on $X$. Then $\left(Q^{*}(t)\right)_{t \geq 0}$ is also a strongly continuous semigroup on $X$ and its generator is $A^{*}$.

To overcome certain difficulties with unboundedness of the generator $A$, we make use of the duality with respect to a pivot space. In general, the idea of (duality with respect to) a pivot space can be described as follows. Having an unbounded closed linear operator $A: D(A) \rightarrow X$ with $D(A) \subset X$ densely, we want to establish a setting where it behaves like a bounded one. One instance of such situation is when we restrict ourselves to the space made out of its domain, but equipped with a graph (or graph-equivalent) norm. It is then reasonable to ask what is the dual of such space. It turns out that it can be represented as a completion of the original space $X$ with a resolvent-induced norm. As the space $X$ is pivotal in the described setting, the name follows. A precise description of such situation can be found in [24, Chapter 2.9] or in [8, Chapter II.5]

The following three propositions from [24, Chapter 2.10] introduce duality with respect to a pivot space (sometimes referred to also as a rigged Hilbert space construction) in the context which we will use later.

Proposition 2.19 Let $A: D(A) \rightarrow X$ be a densely defined operator with $\rho(A) \neq \emptyset$. Then for every $\beta \in \rho(A)$ the space $\left(D(A),\|\cdot\|_{1}\right)$, where

$$
\|z\|_{1}:=\|(\beta I-A) z\|_{X} \quad \forall z \in D(A)
$$

is a Hilbert space denoted $X_{1}$. The norms generated as above for different $\beta \in$ $\rho(A)$ are equivalent to the graph norm. The embedding $X_{1} \subset X$ is continuous. If $Q(t)$ is the semigroup generated by $A$ then $Q(t) \in \mathcal{L}\left(X_{1}\right)$ for every $t \in$ $[0, \infty)$.

For $A$ as in Proposition 2.19 its adjoint $A^{*}$ has the same properties. Thus, we can define the space $X_{1}^{d}:=\left(D\left(A^{*}\right),\|\cdot\|_{1}^{d}\right)$ with the norm

$$
\|z\|_{1}^{d}:=\left\|\left(\bar{\beta} I-A^{*}\right) z\right\|_{X} \quad \forall z \in D\left(A^{*}\right),
$$

where $\beta \in \rho(A)$, and this is also a Hilbert space. 
Proposition 2.20 Let $A: D(A) \rightarrow X$ be a densely defined operator and let $\beta \in \rho(A)$. We denote by $X_{-1}$ the completion of $X$ with respect to the norm

$$
\|z\|_{-1}:=\left\|(\beta I-A)^{-1} z\right\|_{X} \quad \forall z \in X .
$$

Then the norms generated as above for different $\beta \in \rho(A)$ are equivalent (in particular $X_{-1}$ is independent of the choice of $\beta$ ). Moreover, $X_{-1}$ is the dual of $X_{1}^{d}$ with respect to the pivot space $\left(X,\|\cdot\|_{X}\right)$.

The semigroup $(Q(t))_{t \geq 0}$ generated by $A$ has a unique extension $\left(Q_{-1}(t)\right)_{t \geq 0}$ such that $Q_{-1}(t) \in \mathcal{L}\left(X_{-1}\right)$ for every $t \in[0, \infty)$.

Proposition 2.21 Let $A: D(A) \rightarrow X$ be a densely defined operator with $\rho(A) \neq \emptyset, \beta \in \rho(A), X_{1}$ be as in Proposition 2.19 and let $X_{-1}$ be as in Proposition 2.20. Then $A \in \mathcal{L}\left(X_{1}, X\right)$ and it has a unique extension $A_{-1} \in \mathcal{L}\left(X, X_{-1}\right)$. Moreover,

$$
(\beta I-A)^{-1} \in \mathcal{L}\left(X, X_{1}\right), \quad\left(\beta I-A_{-1}\right)^{-1} \in \mathcal{L}\left(X_{-1}, X\right)
$$

(in particular, $\beta \in \rho\left(A_{-1}\right)$ ), and these two operators are unitary.

Remark In the remaining part, we denote the extension $A_{-1}$ and the generator $A$ by the same symbol $A$. The same applies to the semigroup $(Q(t))_{t \geq 0}$.

Consider now a (linear) dynamical system described by the following initial value problem:

$$
\begin{aligned}
\frac{\mathrm{d}}{\mathrm{d} t} z(t) & =A z(t)+B u(t) \\
z(0) & \in X \quad t \in J .
\end{aligned}
$$

where $X$ (called state space) and $U$ (called control space) are the Hilbert spaces; $Z:=L_{l o c}^{1}([0, \infty), X) \cap C\left([0, \infty), X_{-1}\right)$ (called state trajectory space) with $z \in Z$ and $u \in V:=L_{\text {loc }}^{2}([0, \infty), U) \cap C^{1}([0, \infty], U)$ (called control trajectory space $) ; B \in \mathcal{L}\left(U, X_{-1}\right) ; Q(t) \in \mathcal{L}\left(X_{-1}\right)$ for every $t \in J$ is an extension of a semigroup generated by $(A, D(A)), z_{0}:=z(0) \in X$.

The following Definition [24, Definition 4.1.5] is suitable in the context above, namely

Definition 2.22 (Mild solution). The $X_{-1}$-valued function $z$ defined by

$$
z(t):=Q(t) z_{0}+\int_{0}^{t} Q(t-s) B u(s) \mathrm{d} s, \quad t \in J
$$

is called the mild solution of the corresponding differential equation (7).

The two basic types of controllability are given by the following:

Definition 2.23 (approximate controllability). The control process described by (8) is said to be approximately controllable when for any given $z_{0}, x_{T} \in X$ and any $\varepsilon>0$ there exists a control $u$ such that $\left\|z(T)-x_{T}\right\| \leq \varepsilon$, where $z_{0}$ is the initial condition and $u$ is the control.

Definition 2.24 (exact controllability). The control process described by (8) is said to be exactly controllable when $\varepsilon=0$ in Definition 2.23. 
In classical literature (see e.g. [23]), when no rigged Hilbert space construction was used, the following problem was of great importance. Namely, when taking equations (7) as a primary model, its solution must lay in $D(A)$, which is only a dense subset of $X$. That means that the system (7) cannot be exactly controllable. For the same reason, if considering infinite $T$ every approximately controllable system is exactly controllable.

By the use of the rigged Hilbert space construction (called also "a duality with respect to a pivot space") the controllability problem is greatly simplified. First, according to [24, Proposition 4.1.4] every solution to (7) in $X_{-1}$ is a mild solution of (7). Although the converse, in general, still does not have to be true, due to the fact that now $A \in \mathcal{L}\left(X, X_{-1}\right)$ greatly simplifies many considerations.

This, however, comes at a price of the operator $B$ mostly being unbounded from $U$ to $X$. As we would like all the mild solutions (8) to be continuous $X$-valued functions, additional constraints must be put on the operator $B$. This is expressed by the following [24, Definition 4.2.1]:

Definition 2.25 Let $B \in \mathcal{L}\left(U, X_{-1}\right)$ and $\tau \geq 0$. Define the operator $\Phi(\tau)$ as $\Phi(\tau) \in \mathcal{L}\left(L^{2}([0, \infty), U), X_{-1}\right)$,

$$
\Phi(\tau) u:=\int_{0}^{\tau} Q(\tau-\sigma) B u(\sigma) \mathrm{d} \sigma .
$$

The operator $B \in \mathcal{L}\left(U, X_{-1}\right)$ is called an admissible control operator for $(Q(t))_{t \geq 0}$ if for some $\tau>0$ there is $\operatorname{Im} \Phi(\tau) \subset X$.

Remark Note that if $B$ is admissible, then in (9) we integrate in $X_{-1}$ but the integral is in $X$. Also, if the operator $\Phi(\tau)$ is such that $\operatorname{Im} \Phi(\tau) \subset X$ for some $\tau>0$ then for every $t \geq 0$ there is $\Phi(t) \in \mathcal{L}\left(L^{2}([0, \infty), U), X\right)[24$, Proposition 4.2.2]. Obviously, every $B \in \mathcal{L}(U, X)$ is an admissible operator.

The following Proposition [24, Proposition 4.2.5] shows that if $B$ is admissible and $u \in L_{l o c}^{2}([0, \infty), U)$ then the initial value problem $(7)$ has a well-behaved unique solution in $X_{-1}$.

Proposition 2.26 Assume that $B \in \mathcal{L}\left(U, X_{-1}\right)$ is an admissible control operator for $(Q(t))_{t \geq 0}$. Then for every $z_{0} \in X$ and every $u \in L_{l o c}^{2}([0, \infty), U)$ the intial value problem (7) has a unique solution in $X_{-1}$ given by (8) and it satisfies

$$
z \in C([0, \infty), X) \cap H_{l o c}^{1}\left((0, \infty), X_{-1}\right)
$$

\section{Controllability by Schmidt Theorem}

In this section, we present our main findings.

\subsection{Problem statement}

Consider the nonlinear dynamical system with zero initial condition stated by the differential equation in $X_{-1}$ as

$$
\frac{\mathrm{d}}{\mathrm{d} t} z(t)=A z(t)+B u(t)+f(z(t))
$$


where $u \in V=L_{\text {loc }}^{2}([0, \infty), U) \cap C^{\infty}([0, \infty), U), A \in \mathcal{L}\left(X, X_{-1}\right)$ and $B \in$ $\mathcal{L}\left(U, X_{-1}\right)$ is an admissible control operator for $(Q(t))_{t>0}, f: X \rightarrow X$ is a given continuous function. The mild solution of the above initial value problem is

$$
z(t)=\int_{0}^{t} Q(t-s) f(z(s)) \mathrm{d} s+\int_{0}^{t} Q(t-s) B u(s) \mathrm{d} s, \quad t \in J .
$$

The main problem we tackle in this article is to find the conditions under which the dynamical system expressed by (10) is exactly controllable.

\subsection{Step 1}

To show the existence of a solution of problem (10), we build an appropriate integral operator $\Psi: Z \rightarrow Z$ and show that it has a fixed point. Let then

$$
\Psi(z)(t):=\int_{0}^{t} Q(t-s) f(z(s)) \mathrm{d} s+\int_{0}^{t} Q(t-s) B u(s) \mathrm{d} s, \quad t \in J .
$$

In Theorem 2.17 for $z$ to be a unique solution of the Cauchy problem stated there, $z$ has to be also a solution of the integral equation (3). What follows, the integral operator associated with (2) has the form

$$
\Psi(z)(t)=\int_{0}^{t} g(s, z(s)) \mathrm{d} s+\int_{0}^{t} k(s, z(s)) \mathrm{d} s, \quad t \in J .
$$

To show the existence of a fixed point of the operator (11) it is enough to show that appropriate parts of (12) fulfil assumptions of Theorem 2.17. Unfortunately, the obvious choice of functions under integrals in (12), namely $g(s, z(s)):=Q(t-s) f(z(s))$ and $k(s, z(s)):=Q(t-s) B u(s)$ is not possible. The reason for that is that the semigroup $(Q(t))_{t \geq 0}$ is defined for all $t \in[0, T]$, as well as functions $g, k:[0, T] \times X \rightarrow X$ in Theorem 2.17. Hence, for given nonlinearity $f$, we introduce formally two parameter-dependent families of functions

$$
\begin{aligned}
\mathcal{G} & :=\left\{g_{t}:[0, t] \times X \rightarrow X: g_{t}(s, x):=Q(t-s) f(x), t \in[0, T]\right\}, \\
\mathcal{K} & :=\left\{k_{t}:[0, t] \times X \rightarrow X: k_{t}(s, x):=Q(t-s) B u_{x}(s), t \in[0, T]\right\},
\end{aligned}
$$

where the steering trajectory $u_{x}$ is built based on an element $x$ of the state space, as explained below in (15). Taking into account that members $g_{t}$ and $k_{t}$ of both families "work under the integral", the upper limit of which changes in the interval $[0, T]$, Theorem 2.17 cannot be used directly. Instead, we will work it out from other facts.

We make use of the following:

Definition 3.1 Using the notation from Definition 2.22, we define

(a) the Pickard-type [2] operator $L \in \mathcal{L}(Z)$,

$$
L z:=\int_{0}^{\cdot} Q(\cdot-s) z(s) \mathrm{d} s,
$$

(b) the Pickard composition operator $L(t) \in \mathcal{L}\left(Z, X_{-1}\right)$,

$$
L(t) z:=(L z)(t), \quad t \in J,
$$

(c) the nonlinear continuous composition $f z \in Z,(f z)(t):=f(z(t))$ 
With the above definition, the mild solution (10) may be rewritten in the form

$$
z(t)=L(t) f z+L(t) B u .
$$

Let $x_{T} \in X$ be the desired final state. Following a canonical procedure $[18,23]$, we assume exact controllability of the linear system without the nonlinear part $f$, given by Definition 2.22. Then, without loss of generality, we assume that the attainable set $\mathscr{A}_{T}$ is equal to the image of the $L(T) B$ operator, that is,

$$
\begin{aligned}
\mathscr{A}_{T} & :=\left\{x_{T} \in X_{-1}: x_{T}=z(T), u \in V\right\} \\
& =\operatorname{Im}(L(T) B)=\operatorname{Im} \Phi(T)=X .
\end{aligned}
$$

The reason of such approach is to have a possibility to drive the system with nonlinear disturbance $f$ to every point it could attain without such disturbance.

Define a linear and invertible operator $W: V / \operatorname{ker}(L(T) B) \rightarrow X$,

$$
W(u):=L(T) B u .
$$

which has a bounded inverse operator $W^{-1}: X \rightarrow V / \operatorname{ker}(L(T) B)$, with $\left\|W^{-1}\right\| \leq M_{W^{-1}}<\infty$.

As we are interested in exact controllability, let us fix $x_{T} \in X$. We construct a control signal based on this $x_{T}$ by selecting one element

$$
u_{x} \in W^{-1}\left(x_{T}-L(T) f z\right)
$$

which is explicitly related to a trajectory $z$ (with values in $X$ due to admissibility of $B$ ) which system (10) will follow. Substituting control function defined by (15) into operator equation (11) for $t=T$, we obtain

$$
\Psi(z)(T)=L(T) f z+(L(T) B)(L(T) B)^{-1}\left(x_{T}-L(T) f z\right)=x_{T} .
$$

By putting the same control function $u_{x}$ into mild solution (10) we get $z(T)=x_{T}$, what gives $\Psi(z)(T)=z(T)$ and shows that the trajectory end point matches. The only thing left is to show that with the control function $u_{x}$ defined by (15) the operator $\Psi$ defined by (11) has a fixed point in

$$
Z=C([0, \infty), X) \cap H_{l o c}^{1}\left((0, \infty), X_{-1}\right),
$$

(note again that the operator $B$ is assumed to be admissible-Proposition 2.26) what means that there exists such trajectory $z$ of the system (10) which leads it to the given final point $x_{T}$.

\subsection{Step 2}

The existence of a solution to integral equation (12) is equivalent to the existence of a fixed point of the operator (11). We begin with the following:

Proposition 3.2 Let $X$ be a real Hilbert space and we assume that

(H1) $f: X \rightarrow X$ is continuous on $X$ and there exists $M_{f} \in[0, \infty)$ such that $f$ fulfils a one-sided Lipschitz condition, i.e.

$$
\left\langle x_{1}-x_{2}, f\left(x_{1}\right)-f\left(x_{2}\right)\right\rangle \leq M_{f}\left\|x_{1}-x_{2}\right\|^{2} \quad \forall x_{1}, x_{2} \in X, \forall t \in J,
$$


(H2) there exists $M_{g} \in[0, \infty)$ such that for every $t \in J$ the map $g_{t} \in \mathcal{G}$ (i.e. $g_{t}:[0, t] \times X \rightarrow X, g_{t}(s, x):=Q(t-s) f(x)$ ) fulfils a one-sided Lipschitz condition

$$
\left\langle x_{1}-x_{2}, g_{t}\left(s, x_{1}\right)-g_{t}\left(s, x_{2}\right)\right\rangle \leq M_{g}\left\|x_{1}-x_{2}\right\|^{2} \quad \forall x_{1}, x_{2} \in X, \forall s \in[0, t] .
$$

Then for a function $g: J \times X \rightarrow X$ given by $g(t, x):=\frac{\mathrm{d}}{\mathrm{d} t} \int_{0}^{t} g_{t}(s, x) d s$, we have

$$
\left[x_{1}-x_{2}, g\left(t, x_{1}\right)-g\left(t, x_{2}\right)\right]_{-} \leq M_{g}\left\|x_{1}-x_{2}\right\| \quad \forall x_{1}, x_{2} \in X, \forall t \in J .
$$

Proof 1. Fix $x \in X$ and define $G_{x}: J \rightarrow X, G_{x}(t):=\int_{0}^{t} g_{t}(s, x) d s=$ $\int_{0}^{t} Q(t-s) f(x) d s$.

2. With the definition in 1 . it follows that

$$
\begin{aligned}
g(t, x)= & \frac{\mathrm{d}}{\mathrm{d} t} G_{x}(t)=\lim _{h \rightarrow 0} \frac{1}{h}\left(G_{x}(t+h)-G_{x}(t)\right) \\
= & \lim _{h \rightarrow 0} \frac{1}{h}\left(\int_{0}^{t+h} Q(t+h-s) f(x) \mathrm{d} s-\int_{0}^{t} Q(t-s) f(x) \mathrm{d} s\right) \\
= & \lim _{h \rightarrow 0} \frac{1}{h}\left(Q(h) \int_{0}^{t+h} Q(t-s) f(x) \mathrm{d} s-\int_{0}^{t} Q(t-s) f(x) \mathrm{d} s\right) \\
= & \lim _{h \rightarrow 0} \frac{1}{h}\left((Q(h)-I) \int_{0}^{t} Q(t-s) f(x) \mathrm{d} s\right. \\
& \left.+Q(h) \int_{t}^{t+h} Q(t-s) f(x) \mathrm{d} s\right) \\
= & \lim _{h \rightarrow 0}\left(\frac{1}{h}(Q(h)-I) \int_{0}^{t} Q(t-s) f(x) \mathrm{d} s\right. \\
& \left.+\frac{1}{h} \int_{t}^{t+h} Q(t+h-s) f(x) \mathrm{d} s\right) \\
= & A G_{x}(t)+f(x),
\end{aligned}
$$

where the last equality is true provided that both limits on the righthand side exist. We show it below.

3. Fix $t \in J$. Then

$$
\begin{aligned}
& \left\|\frac{1}{h} \int_{t}^{t+h} Q(t+h-s) f(x) \mathrm{d} s-f(x)\right\| \\
& \quad=\left\|\frac{1}{h} \int_{t}^{t+h}(Q(t+h-s) f(x)-f(x)) \mathrm{d} s\right\| \\
& \quad \leq\|Q(t+h-s) f(x)-f(x)\|
\end{aligned}
$$

for some $s \in[t, t+h]$. As $h \rightarrow 0$ there is also $s \rightarrow t$ and by strong continuity of the semigroup $Q(t)$, we have

$$
\lim _{h \rightarrow 0} \frac{1}{h} \int_{t}^{t+h} Q(t+h-s) f(x) d s=f(x), \quad \forall t \in J .
$$


4. For any fixed $x \in X$, hence fixed $f(x) \in X$, by Proposition 2.18 there is

$$
\int_{0}^{t} Q(t-s) f(x) \mathrm{d} s=\int_{0}^{t} Q(\tau) f(x) d \tau \in D(A),
$$

and we have

$$
A \int_{0}^{t} Q(t-s) f(x) \mathrm{d} s=A \int_{0}^{t} Q(\tau) f(x) \mathrm{d} \tau=Q(t) f(x)-f(x) .
$$

In particular, although the integration is formally carried out in $X_{-1}$, the result is in $X$.

5. From points 3 and 4 it follows that

$$
g(t, x)=Q(t) f(x)
$$

is continuous and bounded.

6. Fix $t \in J$ and $x_{1}, x_{2} \in X$. We may write the following estimation:

$$
\begin{aligned}
\left\langle x_{1}-x_{2}, g\left(t, x_{1}\right)-g\left(t, x_{2}\right)\right\rangle & =\left\langle x_{1}-x_{2}, Q(t) f\left(x_{1}\right)-Q(t) f\left(x_{2}\right)\right\rangle \\
& =\left\langle x_{1}-x_{2}, g_{t}\left(0, x_{1}\right)-g_{t}\left(0, x_{2}\right)\right\rangle \leq M_{g}\left\|x_{1}-x_{2}\right\|^{2} .
\end{aligned}
$$

7. As $X$ is a Hilbert space over $\mathbb{R}$, the result of point 6 is equivalent, due to Lemma 2.6, to condition a) of Theorem 2.17.

Before proceeding further, we state a useful lemma.

Lemma 3.3 Let $X$ be a Banach space, $a \in X, \tau<T$ in $\mathbb{R}, J:=[\tau, T]$, $f: J \times X \rightarrow X$ continuous. For $u \in C(J, X)$ define $\Phi: C(J, X) \rightarrow C(J, X)$,

$$
(\Phi u)(t):=a+\int_{\tau}^{t} f(s, u(s)) \mathrm{d} s, \quad \forall t \in J .
$$

If the range $\operatorname{Im} f \subseteq S \subseteq X$ then $(\Phi u)(t) \in a+(T-\tau) \operatorname{cl} \operatorname{conv}(S \cup\{0\})$.

Proof 1. Fix $u \in C(J, X)$. We have

$$
(\Phi u)(t) \approx a+\sum_{k=1}^{n}\left(t_{k}-t_{k-1}\right) f\left(\tau_{k}, u\left(\tau_{k}\right)\right)
$$

where $\tau=t_{0}<t_{1}<t_{2}<\cdots<t_{n}=t$.

2. Rewriting above, we get

$$
(\Phi u)(t) \approx a+(T-\tau)\left[\sum_{k=1}^{n} \frac{t_{k}-t_{k-1}}{T-\tau} f\left(\tau_{k}, u\left(\tau_{k}\right)\right)+\frac{T-t}{T-\tau} \Theta\right],
$$

where $\Theta \in S \cup\{0\}$.

3. As $\sum_{k=1}^{n} \frac{t_{k}-t_{k-1}}{T-\tau}+\frac{T-t}{T-\tau}=1$, there is

$$
\left[\sum_{k=1}^{n} \frac{t_{k}-t_{k-1}}{T-\tau} f\left(\tau_{k}, u\left(\tau_{k}\right)\right)+\frac{T-t}{T-\tau} \Theta\right] \in \operatorname{conv}(S \cup\{0\}) \subseteq \operatorname{clconv}(S \cup\{0\}) .
$$

4. As integral is a limit to the Riemann sums, each of which belongs to $\operatorname{conv}(S \cup\{0\})$, the integral itself belong to $\operatorname{cl} \operatorname{conv}(S \cup\{0\})$, i.e.

$$
(\Phi u)(t) \in a+(T-\tau) \operatorname{cl} \operatorname{conv}(S \cup\{0\}) .
$$


Let us now focus on assumption (b) of Theorem 2.17. We can relate it to our controllability setting by the following:

Proposition 3.4 Using previously defined notation, if

(H3) the operator $B \in \mathcal{L}(U, X)$ (hence, as bounded from $U$ to $X$, it is an admissible control operator for $\left.(Q(t))_{t>0}\right)$ and the linear system (8) is exactly controllable to the space $X=(L(T) B)$,

(H4) the operator $W: V / \operatorname{ker}(L(T) B) \rightarrow X, W(u):=L(T) B u$ has a bounded inverse operator $W^{-1}$,

(H5) the space $X$ is ordered by a normal wedge $C$ and the operator $W^{-1}$ is such that for every $y \in X$ the function $f:[0, t] \rightarrow X$,

$$
f(s):=Q(t-s) B W^{-1}(y)(s)
$$

is convex,

(H6) there exists $M_{w} \in[0, \infty)$ such that

$$
\alpha\left(W^{-1}(D)(t)\right) \leq M_{w} \alpha(D) \quad \forall t \in J, \forall D \subset X, D \text { bounded, }
$$

(Hr) there exists $M_{w}^{\prime} \in[0, \infty)$ such that

$$
\alpha\left(W^{-1}\left(D^{\prime}\right)(t)\right) \leq M_{w}^{\prime} \alpha(D) \quad \forall t \in J, \forall D \subset X, D \text { bounded, }
$$

where $D^{\prime}:=\left\{y \in X: W^{-1}(y)=\frac{\mathrm{d}}{\mathrm{d} s} W^{-1}(x), x \in D\right\}$,

then for a function $k: J \times X \rightarrow X$ given by $k(t, x):=\frac{\mathrm{d}}{\mathrm{d} t} \int_{0}^{t} Q(t-$ $s) B W^{-1}(x)(s) \mathrm{d} s$, we have

$$
\alpha(k(J, D)) \leq M_{k} \alpha(D) \quad \forall D \subseteq X, D \text { bounded } .
$$

Proof 1. From Definition 2.8 for every $s \in[0, T]$ and every bounded $D \subset$ $X$, we have

$$
\begin{aligned}
\alpha\left(W^{-1}(D)(s)\right)= & \inf \left\{\delta(s) \geq 0: W^{-1}(D)(s) \subset \bigcup_{i=1}^{n} \Sigma_{i}(s)\right. \\
& \left.\operatorname{diam} \Sigma_{i}(s) \leq \delta(s), \Sigma_{i}(s) \subset U, i \in\{1, \ldots, n\} ; n \in \mathbb{N}\right\},
\end{aligned}
$$

where $\Sigma_{i} \subset V / \operatorname{ker}(L(T) B)=L_{l o c}^{2}([0, \infty), U) \cap C^{\infty}([0, \infty), U) /$ $\operatorname{ker}(L(T) B)$.

2. Further, for every $\tau \in[0, T]$, we have

$$
\begin{aligned}
\alpha\left(Q(\tau) B W^{-1}(D)(s)\right)= & \inf \left\{\delta^{\prime}(s) \geq 0: Q(\tau) B W^{-1}(D)(s) \subset \bigcup_{i=1}^{n} Q(\tau) B \Sigma_{i}(s) ;\right. \\
& \left.\operatorname{diam} Q(\tau) B \Sigma_{i}(s) \leq \delta^{\prime}(s)\right\}
\end{aligned}
$$

and

$$
\operatorname{diam}\left(\Sigma_{i}(s)\right)=\sup _{u(s), v(s) \in \Sigma_{i}(s)}\|u(s)-v(s)\| .
$$

Let us fix $u(s), v(s) \in \Sigma_{i}(s)$ and let $x(s):=Q(\tau) B u(s), y(s):=$ $Q(\tau) B v(s)$. We then have $x(s), y(s) \in Q(\tau) B \Sigma_{i}(s)$ and $\|x(s)-y(s)\|=\|Q(\tau) B(u(s)-v(s))\| \leq\|Q(\tau) B\|\|u(s)-v(s)\|$. 
Hence, $\operatorname{diam}\left(Q(\tau) B \Sigma_{i}(s)\right) \leq M_{q}\|B\| \operatorname{diam}\left(\Sigma_{i}(s)\right)$, where $M_{q}:=$ $\max _{t \in J}\|Q(t)\|$. Using point 1, we may now write

$$
\operatorname{diam}\left(Q(\tau) B \Sigma_{i}(s)\right) \leq \delta^{\prime}(s) \leq M_{q}\|B\| \operatorname{diam}\left(\Sigma_{i}(s)\right) \leq M_{q}\|B\| \delta(s)
$$

for suitably chosen $\delta^{\prime}(s), \delta(s)$. Passing to infimum we get the estimation

$$
\alpha\left(Q(\tau) B W^{-1}(D)(s)\right) \leq M_{q}\|B\| \alpha\left(W^{-1}(D)(s)\right)
$$

for every $s, \tau \in J$ and every bounded $D \subset X$.

3. Using now assumption $(H 6)$, we obtain

$$
\alpha\left(Q(\tau) B W^{-1}(D)(s)\right) \leq M_{q}\|B\| M_{w} \alpha(D)
$$

for every $s, \tau \in J$ and every bounded $D \subset X$.

4. Preparing the ground for the Ambrosetti Theorem 2.15 let $J_{t}:=[0, t] \subset$ $[0, T]$ and define a family of operators indexed by the elements of $D \subset X$, namely

$$
\mathcal{F}_{t}:=\left\{Q(t-\cdot) B W^{-1}(y)(\cdot)\right\}_{y \in D} \subset C\left(J_{t}, X\right) .
$$

We will show that for every $t \in J$ and every bounded $D \subset X$ the family $\mathcal{F}_{t} \subset C\left(J_{t}, X\right)$ is equicontinuous. For that purpose note first that the operator $W^{-1}$ is a bounded and linear operator, hence it is continuous on $X$.

Now fix $t \in J$ and define a function $\varphi_{t}: J_{t} \times X \rightarrow X$,

$$
\varphi_{t}(s, y):=Q(t-s) B W^{-1}(y)(s) .
$$

We will show that $\varphi_{t}$ is continuous on the product $J_{t} \times X$. Fix $y \in X$ and let $s_{1}, s_{2} \in J_{t}$ be such that $s_{1}+\tau=s_{2}, \tau>0$. We then have

$$
\begin{aligned}
\| Q( & \left.t-s_{1}\right) B W^{-1}(y)\left(s_{1}\right)-Q\left(t-s_{2}\right) B W^{-1}(y)\left(s_{2}\right) \| \\
= & \left.\| Q\left(t-s_{2}+\tau\right)\right) B W^{-1}(y)\left(s_{2}-\tau\right)-Q\left(t-s_{2}\right) B W^{-1}(y)\left(s_{2}\right) \| \\
= & \left\|Q\left(t-s_{2}\right) Q(\tau) B W^{-1}(y)\left(s_{2}-\tau\right)-Q\left(t-s_{2}\right) B W^{-1}(y)\left(s_{2}\right)\right\| \\
= & \| Q\left(t-s_{2}\right)\left(Q(\tau) B W^{-1}(y)\left(s_{2}-\tau\right)-Q(\tau) B W^{-1}(y)\left(s_{2}\right)\right. \\
& \left.+Q(\tau) B W^{-1}(y)\left(s_{2}\right)-B W^{-1}(y)\left(s_{2}\right)\right) \| \\
\leq & \left\|Q\left(t-s_{2}\right)\right\|\|Q(\tau)\|\left\|B W^{-1}(y)\left(s_{2}-\tau\right)-B W^{-1}(y)\left(s_{2}\right)\right\| \\
& +\left\|Q\left(t-s_{2}\right)\right\|\left\|Q(\tau) B W^{-1}(y)\left(s_{2}\right)-B W^{-1}(y)\left(s_{2}\right)\right\|,
\end{aligned}
$$

where the last part tends to 0 with $s_{1} \rightarrow s_{2}$, that is with $\tau \rightarrow 0$. This follows from the continuity of $W^{-1}(y)$ on $J_{t}$ and strong continuity of the semigroup $Q$. Now joint continuity of $\varphi_{t}$ follows from linearity and continuity of $W^{-1}$ on $X$ and the decomposition

$$
\varphi_{t}(s, y)-\varphi_{t}(\tau, z)=\varphi_{t}(s, y)-\varphi_{t}(s, z)+\varphi_{t}(s, z)-\varphi_{t}(\tau, z),
$$

where $(\tau, z) \rightarrow(s, y)$.

From continuity of $\varphi_{t}$ it follows that for every bounded $D \subset X$ the set $\varphi_{t}\left(J_{t}, D\right) \subset X$ remains bounded, i.e. there exists such $r<\infty$ that 
$\varphi_{t} \subset B(0, r)$, a zero-centred ball with a finite radius $r$. Defining, for a given bounded $D \subset X$, the set

$$
\mathcal{F}_{t}(s):=\left\{\theta(s): \theta \in \mathcal{F}_{t}\right\}
$$

we have

$$
\mathcal{F}_{t}(s)=\bigcup_{y \in D} \varphi_{t}(s, y) \subset B(0, r)
$$

for suitable $r<\infty$. By $(H 5)$ and Theorem 2.14 it follows that the collection of continuous mappings $\mathcal{F}_{t}$ is equicontinuous for every $t \in J$ and every bounded $D \subset X$.

5. Fix bounded $D \subset X$. From the Ambrosetti Theorem 2.15 and point 4 we have

$$
\alpha\left(\mathcal{F}_{t}\right)=\sup _{s \in J_{t}} \alpha\left(\mathcal{F}_{t}(s)\right)=\alpha\left(\mathcal{F}_{t}\left(J_{t}\right)\right) \quad \forall t \in J
$$

where $\mathcal{F}_{t}\left(J_{t}\right)=\bigcup_{s \in J_{t}} \mathcal{F}_{t}(s)$. Point 3 gives

$\alpha\left(\mathcal{F}_{t}(s)\right)=\alpha\left(Q(t-s) B W^{-1}(D)(s)\right) \leq M_{q}\|B\| M_{w} \alpha(D) \quad \forall t \in J \forall s \in J_{t}$.

In consequence, we have

$$
\sup _{s \in J_{t}} \alpha\left(\mathcal{F}_{t}(s)\right)=\alpha\left(\mathcal{F}_{t}\left(J_{t}\right)\right) \leq M_{q}\|B\| M_{w} \alpha(D) \quad \forall t \in J
$$

Note that for every $t \in J$ and every $y \in D$ there is $\varphi_{t}(s, y) \in \mathcal{F}_{t}(s)$. Hence, $\varphi_{t}\left(J_{t}, D\right)=\left\{\varphi(s, y): s \in J_{t}, y \in D\right\}=\mathcal{F}_{t}\left(J_{t}\right)$ and for every $t \in J$ and every bounded $D \subset X$, we get

$$
\alpha\left(\varphi_{t}\left(J_{t}, D\right)\right) \leq M_{k} \alpha(D)
$$

hence, for every $t \in J$ the mapping $\varphi_{t}: J_{t} \times X$ is condensing with constant $M_{k}:=M_{q}\|B\| M_{w}$. 
6. Define $K: J \times X \rightarrow X, K(t, x):=\int_{0}^{t} \varphi_{t}(s, x) \mathrm{d} s=\int_{0}^{t} Q(t-$ $s) B W^{-1}(x)(s) \mathrm{d} s$. Fix $t \in J$ and $x \in X$, then

$$
\begin{aligned}
k(t, x)= & \frac{\mathrm{d}}{\mathrm{d} t} K(t, x)=\lim _{h \rightarrow 0} \frac{1}{h}(K(t+h, x)-K(t, x)) \\
= & \lim _{h \rightarrow 0} \frac{1}{h}\left(\int_{0}^{t} Q(t+h-s) B W^{-1}(x)(s) \mathrm{d} s\right. \\
& -\int_{0}^{t} Q(t-s) B W^{-1}(x)(s) \mathrm{d} s \\
& \left.+\int_{t}^{t+h} Q(t+h-s) B W^{-1}(x)(s) \mathrm{d} s\right) \\
= & \lim _{h \rightarrow 0}\left(\frac{1}{h}(Q(h)-I) \int_{0}^{t} Q(t-s) B W^{-1}(x)(s)\right) \mathrm{d} s \\
& \left.+\frac{1}{h} \int_{t}^{t+h} Q(t+h-s) B W^{-1}(x)(s) d s\right) \\
= & A K(t, x)+B W^{-1}(x)(t)+\int_{0}^{t} Q(t-s) B \frac{\mathrm{d}}{\mathrm{d} s} W^{-1}(x)(s) \mathrm{d} s \\
= & Q(t) B W^{-1}(x)(0)+L(t) B u_{x}^{\prime},
\end{aligned}
$$

provided that appropriate limits exist. We show it below.

7. Consider again the function $\varphi_{t}$ defined in (18). Calculating its time derivative at $s \in[0, t]$ we obtain

$$
\frac{\mathrm{d}}{s} \varphi_{t}(s, x)=-A Q(t-s) B W^{-1}(x)(s)+Q(t) B \frac{\mathrm{d}}{\mathrm{d} s} W^{-1}(x)(s) .
$$

Initially, the above result can be found either by elementary limit calculation or one can use the result in [8, Lemma B.16]. Here, both parts on the right-hand side exist, although for the sake of clarity we skip all the routine limit considerations in the argument of the generator $A$ leading to application of its extension - for more details see Proposition 2.21 and [24, Proposition 2.10.3]. Note also, that by assumption the function

$$
u_{x}^{\prime}:[0, t] \rightarrow U, \quad u_{x}^{\prime}:=\frac{\mathrm{d}}{\mathrm{d} s} W^{-1}(x)
$$

exists for all $x \in X$ and is continuous.

We also have the following:

$$
\begin{gathered}
B W^{-1}(x)(t)-Q(t) B W^{-1}(x)(0)=\varphi_{t}(t, x)-\varphi_{t}(0, x)=\int_{0}^{t} \frac{\mathrm{d}}{\mathrm{d} s} \varphi_{t}(s, x) \mathrm{d} s \\
=-\int_{0}^{t} A \varphi_{t}(s, x) \mathrm{d} s+L(t) B u_{x}^{\prime}=-A \int_{0}^{t} \varphi_{t}(s, x) \mathrm{d} s+L(t) B u_{x}^{\prime},
\end{gathered}
$$


where due to Proposition 2.18, we can move from the extension to the original generator $A$. In consequence,

$$
\begin{aligned}
A K(t, x) & =A \int_{0}^{t} \varphi_{t}(s, x) \mathrm{d} s=A \int_{0}^{t} Q(t-s) B W^{-1}(x)(s) \mathrm{d} s \\
& =Q(t) B W^{-1}(x)(0)-B W^{-1}(x)(t)+L(t) B u_{x}^{\prime} .
\end{aligned}
$$

8. To finish the proof of (21), consider the following estimation:

$$
\begin{aligned}
& \left\|\frac{1}{h} \int_{t}^{t+h} Q(t+h-s) B W^{-1}(x)(s) \mathrm{d} s-B W^{-1}(x)(t)\right\| \\
& =\frac{1}{h}\left\|\int_{t}^{t+h}\left(Q(t+h-s) B W^{-1}(x)(s)-B W^{-1}(x)(t)\right) \mathrm{d} s\right\| \\
& \leq\left\|Q(t+h-s) B W^{-1}(x)(s)-Q(t+h-s) B W^{-1}(x)(t)\right\| \\
& \quad+\left\|Q(t+h-s) B W^{-1}(x)(t)-B W^{-1}(x)(t)\right\|,
\end{aligned}
$$

for some $s \in[t, t+h]$. Taking the limit as $h \rightarrow 0$ there is also $s \rightarrow t$ and due the continuity of $t \mapsto Q(t)$ and continuity of $W^{-1}(x)$ above tends to zero and we obtain

$$
\lim _{h \rightarrow 0} \frac{1}{h} \int_{t}^{t+h} Q(t+h-s) B W^{-1}(x)(s) \mathrm{d} s=B W^{-1}(x)(t) .
$$

Combining now this result and the one of point 7, we obtain (21).

9. Fix bounded $D \subset X$. We have

$$
k(t, x)=Q(t) B W^{-1}(x)(0)+L(t) B u_{x}^{\prime}=k_{t}(0, x)+L(t) B u_{x}^{\prime}
$$

and

$$
k(J, D)=\bigcup_{x \in D} \bigcup_{t \in J} k(t, x)
$$

Note that for every $t \in J$ and every $x \in D$ there is $Q(t) B W^{-1}(x)(0) \in$ $\mathcal{F}_{t}(0)$ with $\mathcal{F}_{t}$ defined for the same index set $D$.

Due to the fact that $W$ is an injection, for every $x \in X$, there exists a unique $y \in X$ such that

$$
W^{-1}(y)=u_{x}^{\prime}=\frac{\mathrm{d}}{\mathrm{d} s} W^{-1}(x) \in V / \operatorname{ker} L(T) B
$$

and $\left\|u_{x}^{\prime}\right\|=\left\|W^{-1}(y)\right\|<\infty$. In consequence, for every bounded $D \subset X$ the set

$$
D^{\prime}:=\left\{y \in X: W^{-1}(y)=u_{x}^{\prime}=\frac{\mathrm{d}}{\mathrm{d} s} W^{-1}(x), x \in D\right\}
$$

is unique.

Define, similar to point 4 , the equicontinuous family of operators $\mathcal{F}_{t}^{\prime}:=\left\{Q(t-\cdot) B W^{-1}(y)(\cdot)\right\}_{y \in D^{\prime}}=\left\{Q(t-\cdot) B u_{x}^{\prime}\right\}_{x \in D} \subset C\left(J_{t}, X\right)$. 
which may be regarded as indexed by elements of either $D^{\prime}$ or $D$. Using the assumption $(H 7)$ and following the same procedure which led to (20), we have

$$
\alpha\left(\mathcal{F}_{t}^{\prime}\left(J_{t}\right)\right) \leq M_{q}\|B\| M_{w}^{\prime} \alpha(D)
$$

As the range of the function $[0, t] \ni s \mapsto Q(t-s) B u_{x}^{\prime}(s) \in X$ is contined in $\mathcal{F}_{t}^{\prime}\left(J_{t}\right)$, according to Lemma 3.3 there is

$$
L(t) B u_{x}^{\prime} \in T \operatorname{cl} \operatorname{conv}\left(\mathcal{F}_{t}^{\prime}\left(J_{t}\right) \cup\{0\}\right) .
$$

Define now a collection of operators $\mathcal{P}:=\{k(\cdot, x)\}_{x \in D}$, where each member acts from $J$ to $X$. From point 4 and above considerations, we see that $\mathcal{P}$ is in fact a collection of bounded operators, indexed again by elements of $D \subset X$. With the same reasoning as in point 4 we see that $\mathcal{P}$ is an equicontinuous set and, by Ambrosetti Theorem 2.15, we have

$$
\alpha(\mathcal{P})=\sup _{t \in J} \alpha(\mathcal{P}(t))=\alpha(\mathcal{P}(J)) \quad \forall t \in J
$$

Due to the definition of $\mathcal{P}$, we have $k(J, D)=\mathcal{P}(J)$ and

$$
\begin{aligned}
& \mathcal{P}(t)=\bigcup_{x \in D} Q(t) B W^{-1}(x)(0)+L(t) B u_{x}^{\prime} \subset \mathcal{F}_{t}\left(J_{t}\right) \cup \\
& T \text { cl conv }\left(\mathcal{F}_{t}^{\prime}\left(J_{t}\right) \cup\{0\}\right)
\end{aligned}
$$

for every $t \in J$. From (20), (24) and (25) and Theorems 2.9 and 2.10 it now follows that

$$
\begin{aligned}
\alpha(k(J, D)) & =\alpha(P(J)) \leq \alpha\left(\mathcal{F}_{t}\left(J_{t}\right) \cup T \operatorname{cl} \operatorname{conv}\left(\mathcal{F}_{t}^{\prime}\left(J_{t}\right) \cup\{0\}\right)\right) \\
& \leq \max \left\{M_{w}, M_{w}^{\prime}\right\} T M_{q}\|B\| \alpha(D),
\end{aligned}
$$

and function $k$ is condensing.

Based on Propositions 3.2 and 3.4, we may state the main Theorem of this article which gives sufficient conditions for the existence of a solution to integral equation (12). This is equivalent to the existence of a fixed point of the operator (11) and results in exact controllability of system (10).

Theorem 3.5 Assume $(H 1)-(H 7)$. Then a dynamical system with mild solution given by (10) is exactly controllable to the space $\operatorname{Im}(L(T) B)=X$, with trajectory $z \in C([0, \infty), X) \cap \mathcal{H}_{\text {loc }}^{1}\left((0, \infty), X_{-1}\right)$.

Proof The proof follows immediately from the reasoning in Step 1 section, Propositions 3.2, 3.4 and application of Theorem 2.17.

\section{Example}

Let us take an example similar to the one chosen in [12], but with an emphasis put on nonlinearity $f$. Consider a one-dimensional real non-homogeneous transport partial differential equation with nonlinear part given by

$$
\begin{aligned}
\frac{\partial}{\partial t} z(t, \xi) & =\frac{\partial}{\partial \xi} z(t, \xi)+m(\xi) u(t, \xi)+f(z(t, \xi)), \\
z(0, \xi) & =0 \in X
\end{aligned}
$$


where spatial coordinate $\xi \in[0,1]$, time coordinate $t \in J:=[0, T]$, the state space $X$ and control space $U$ be $L^{2}(0,1)$. In other words, we consider the mapping $z$ defined on the Cartesian product $[0, T] \times[0,1]$ as the state trajectory which for every $t \in J$ takes value $z(t)$, which is a mapping of $L^{2}$ class from the interval $[0,1]$ to $\mathbb{R}$.

Let $A: D(A) \rightarrow X, D(A) \subset X$ densely, be a generator of a vanishing (or nilpotent) left shift semigroup [24, Example 2.3.8], defined as a spatial differentiation operator

$$
A x:=\frac{d}{d \xi} x, \quad D(A):=\left\{x \in H^{1}(0,1): x(1)=0\right\},
$$

where $\mathcal{H}^{1}(0,1)$ is the Sobolev space of all $L^{2}(0,1)$ functions for which its first derivative is also square integrable [9, Definition 5.2.2]. The semigroup $(Q(t))_{t \geq 0}$ is explicitly given by

$$
(Q(t) x)(\xi):= \begin{cases}x(\xi+t) & \text { if } \xi \in[0,1], \xi+t \leq 1, \\ 0 & \text { if } \xi \in[0,1], \xi+t>1,\end{cases}
$$

where we take $t \in J$. The semigroup $(Q(t))_{t \geq 0}$ is not compact on $X$, but $\alpha(Q(t) D) \leq 2 \alpha(D)$ for every bounded set $D \subset X$, making it a condensing operator. Note also, that the semigroup $(Q(t))_{t \geq 0}$ is contractive.

Define the control operator $B \in \mathcal{L}(U, X)$ appropriately as

$$
B u(t)(\xi):=m(\xi) u(t)(\xi),
$$

for every $t \in J$ and $\xi \in[0,1]$ where $m(\xi)$ provides a spatial distribution of control.

It is known that Hilbert spaces $L^{2}(0,1)$ and $l^{2}$ are isometrically isomorphic [14]. Let $P: L^{2}(0,1) \rightarrow l^{2}$ be such isometric isomorphism. Define also continuous $\varphi: \mathbb{R} \rightarrow \mathbb{R}$ as

$$
\varphi(\xi):= \begin{cases}0 & \text { if } \xi<0, \\ -\sqrt{\xi} & \text { if } \xi \in[0,1] \\ -1 & \text { if } \xi>1\end{cases}
$$

and $\rho: l^{2} \rightarrow l^{2}, \rho(\alpha)=\rho\left(\left(\alpha^{1}, \alpha^{2}, \alpha^{3}, \ldots\right)\right):=\left(\varphi\left(\alpha^{1}\right), \frac{1}{2} \varphi\left(\alpha^{2}\right), \frac{1}{3} \varphi\left(\alpha^{3}\right), \ldots\right)$. Let now the nonlinearity $f: X \rightarrow X$ be given by

$$
f(x):=\left(P^{-1} \rho P\right)(x) .
$$

The dissipativity condition from assumption $(H 1)$ in Proposition 3.2, that is,

$$
\langle x-y, f(x)-f(y)\rangle \leq M_{f}\|x-y\|^{2} \quad \forall x, y \in X
$$

is equivalent to

$$
\langle P x-P y, \rho P x-\rho P y\rangle \leq M_{f}\|P x-P y\|^{2} \quad \forall x, y \in X .
$$

We will show that $f$ does not fulfil Lipschitz condition

$$
\exists_{M_{f}<\infty} \forall_{x, y \in L^{2}(0,1)} \quad\|f(x)-f(y)\| \leq M_{f}\|x-y\|,
$$

which using the definition of $f$, is equivalent to

$$
\exists_{M_{f}<\infty} \forall_{x, y \in L^{2}(0,1)} \quad\|\rho P x-\rho P y\| \leq M_{f}\|P x-P y\| .
$$


Indeed, fix $y=0$ and such sequence $\left(x_{m}\right)_{m \in \mathbb{N}}$ of elements of $L^{2}(0,1)$ that $\left(\alpha_{m}\right)_{m \in \mathbb{N}}:=\left(P x_{m}\right)_{m \in \mathbb{N}}$ and $\alpha_{m}=\left(\frac{1}{m}, 0,0, \ldots\right), m \in \mathbb{N}$. Note that $\alpha_{m} \rightarrow$ $0=P y$ as $m \rightarrow \infty$. We then have

$$
\frac{\left\|\rho\left(P x_{m}\right)\right\|}{\left\|P x_{m}\right\|}=\frac{\left\|\rho\left(\alpha_{m}\right)\right\|}{\left\|\alpha_{m}\right\|}=\frac{\sqrt{\varphi^{2}\left(\alpha^{1}\right)}}{\sqrt{\frac{1}{m^{2}}}}=\frac{\sqrt{\frac{1}{m}}}{\frac{1}{m}}=\sqrt{m},
$$

and as $\sqrt{m} \rightarrow \infty$ as $m \rightarrow \infty$, we see that Lipschitz condition (30) cannot be fulfilled at $y=0$.

It remains to show that $f$ fulfils condition (28). Fix $P x=\alpha$ and $P y=\beta$. We have

$$
\langle\alpha-\beta, \rho(\alpha)-\rho(\beta)\rangle=\sum_{i=1}^{\infty}\left(\alpha^{i}-\beta^{i}\right)\left(\varphi\left(\alpha^{i}\right)-\varphi\left(\beta^{i}\right)\right) \leq 0,
$$

because due to monotonicity of $\varphi$, the element $\left(\alpha^{i}-\beta^{i}\right)\left(\varphi\left(\alpha^{i}\right)-\varphi\left(\beta^{i}\right)\right) \leq 0$ for every $i \in \mathbb{N}$. Hence, it is enough to take any positive $M_{f}$ in (28) and the condition is met. Note also that with the semigroup Definition (27) assumption (H2) is equivalent to (28).

Note also that $f$ is uniformly bounded. This follows from the fact that

$$
\|f(x)\|=\|\rho(P x)\| \quad \forall x \in X
$$

and

$$
\|\rho(\alpha)\|^{2}=\sum_{n=1}^{\infty}\left[\frac{1}{n} \varphi\left(\alpha^{n}\right)\right]^{2} \leq \sum_{n=1}^{\infty} \frac{1}{n^{2}}<\infty .
$$

\section{Conclusions}

In this article, we showed new results in establishing sufficient conditions for controllability of particular types of dynamical systems. Our results expand the results found in [18], where the authors use Nussbaum fixed point theorem. In particular, we did not impose any compactness condition on the semigroup, instead we used its condensing property. This is a considerably weaker assumption than the one taken in the above mentioned work.

The second improvement in comparison to the present state of literature is that we did not assume that the nonlinearity is Lipschitz. The price paid for that is that we used an existence result initially intended for the initial value problem, not formulated in a fixed point form. The authors are not aware whether there exists a similar fixed point theorem.

Our result can be expanded to incorporate phenomena such as impulsive behaviour or nonlocal conditions in a way similar to [12]. Note, however, that the assumptions of the Schmidt theorem are in a sense weaker than the demands of the Mönch's condition used be the authors of [12]. 


\section{Acknowledgements}

This project has received funding from the European Union's Horizon 2020 research and innovation programme under the Marie Skłodowska-Curie Grant Agreement No. 700833.

Open Access. This article is distributed under the terms of the Creative Commons Attribution 4.0 International License (http://creativecommons.org/licenses/ by/4.0/), which permits unrestricted use, distribution, and reproduction in any medium, provided you give appropriate credit to the original author(s) and the source, provide a link to the Creative Commons license, and indicate if changes were made.

\section{References}

[1] Ambrosetti, A.: Un teorema di esistenza per la equazioni differenziali negli spazi di Banach. Rend. Sem. Univ. Padova 39, 349-361 (1967)

[2] Appell, J.: Measures of noncompactness, condensing operators and fixed points: an application-oriented survey. Fixed Point Theory 6, 157-229 (2005)

[3] Balachandran, K., Dauer, J.P.: Controllability of nonlinear systems in Banach spaces: A survey. J. Optim. Theory Appl. 115, 7-28 (2002)

[4] Banaś, J., Goebel, K.: Measures of noncompactness in Banach spaces, Lecture Notes in Pure and Applied Mathematics. CRC Press, Boca Raton (1980)

[5] Breckner, W.W.: Equicontinuous families of generalized convex mappings. Mathematica (Cluj) 26(49), 9-20 (1984)

[6] Breckner, W.W., Trif, T.: Equicontinuity and Hölder equicontinuity of families of generalized convex mappings. N. Z. J. Math. 28, 155-170 (1999)

[7] Darbo, G.: Punti uniti in trasformazioni a codominio non compatto. Rend. Sem. Mat. Univ. Padova 24, 84-92 (1955)

[8] Engel, K.-J., Nagel, R.: One-Parameter Semigroup for Linear Evolution Equations, Graduate Texts in Mathematics, vol. 194. Springer, Berlin (2000)

[9] Evans, L.C.: Partial Differential Equations, Graduate Studies in Mathematics, vol. 19. American Mathematical Society, Providence (2002)

[10] Kosmol, P., Schill, W., Wriedt, M.: Der Satz von Banach-Steinhaus für konvexe Operatoren. Arch. Math. 33, 564-569 (1979)

[11] Kuratowski, C.: Sur les espaces complèts. Fund. Math. 15, 301-309 (1930)

[12] Machado, J.A., Ravichandran, C., Rivero, M., Trujillo, J.J.: Controllability results for impulsive mixed-type functional integro-differential evolution equations with nonlocal conditions. Fixed Point Theory Appl. 66, 1-16 (2013)

[13] Mönch, H.: Boundary value problem for nonlinear ordinary differential equations of second order in Banach spaces. Nonlinear Anal. 4, 985-999 (1980)

[14] Muscat, J.: Functional Analysis: An introduction to metric spaces, Hilbert spaces. Springer, New York (2014)

[15] Nussbaum, R.P.: The fixed point index and asymptotic fixed point theorems for K-set contractions. Bull. Am. Math. Soc. 18, 490-495 (1969) 
[16] Obukhovski, V., Zecca, P.: Controllability for systems governed by semilinear differential inclusions in a Banach space with a noncompact semigroup. Nonlinear Anal. 70, 3424-3436 (2009)

[17] Pazy, A.: Semigroups of linear operators and applications to partial differential equations. Springer, New York (1983)

[18] Quinn, M.D., Carmichael, N.: An approach to non-linear control problems using fixed-point methods, degree theory and pseudo-inverses. Numer. Funct. Anal. Optim. 7, 197-219 (1985)

[19] Rudin, W.: Functional Analysis, 2nd edn. McGraw-Hill, New York (1991)

[20] Sakthivel, R., Choi, Q.H.: A study on controllability of semilinear integrodifferential systems in Banach spaces. Comput. Math. Appl. 47, 519-527 (2004)

[21] Schmidt, S.: Existenzsätze für gewöhnliche differentialgleichungen in Banachräumen. Funkcial. Ekvac. 35, 199-222 (1992)

[22] Shaefer, H.: Uber die methode der a priori schranken. Math. Ann. 129, 415-416 (1955)

[23] Triggiani, R.: On the lack of exact controllability for mild solutions in Banach spaces. J. Math. Anal. Appl. 50, 438-446 (1975)

[24] Tucsnak, M., Weiss, G.: Observation and Control for Operator Semigroups. Birkhäuser Verlag AG, Basel (2009)

[25] Volkmann, P.: Cinq cours sur les équations différentielles dans les espaces de Banach, Topological Methods in Differential Equations and Inclusions. In: Granas, A., Frigon, M., Sabidussi, G. (eds.) NATO ASI Series, vol. 472. Springer, Amsterdam, pp. 501-520 (1995)

[26] Zawiski, R.: On controllability and measures of noncompactness, Proceedings of the 10th International Conference on Mathematical Problems in Engineering, Aerospace and Sciences: ICNPAA 2014 (Seenith Sivasundaram, ed.), vol. 1637, pp. 1241-1246 (2014)

Radosław Zawiski

School of Mathematics

University of Leeds

Leeds LS2 9JT

UK

e-mail: R.Zawiski@leeds.ac.uk 